\title{
Formulation and Characterization of Glutathione-Loaded Bioadhesive Hydrogel for Ocular Delivery
}

\author{
Guanyu Chen, Teresa Hawke, Diana Wong, Jonathon Hwang, Katarina Jeftic, Min Hao Zhan and Jingyuan Wen*
}

School of Pharmacy, Faculty of Medical and Health Science, University of Auckland, New Zealand

*Corresponding author: Jingyuan Wen, School of Pharmacy, Faculty of Medical and Health Science, University of Auckland, New Zealand, Tel: +6493737599; E-mail: j.wen@auckland.ac.nz

Received February 11, 2019; Accepted April 16, 2019; Published April 24, 2019

\begin{abstract}
Glutathione may prevent age-related, oxidative damage to ocular tissues but has poor corneal penetration. Hydrogel formulations were investigated to determine an optimized ocular delivery system. The rheology and texture profile of formulations were investigated at $25^{\circ} \mathrm{C}$. The $1 \% \mathrm{w} / \mathrm{v}$ glutathione was incorporated into systems demonstrating desirable characteristics for ocular vehicles and physical characteristics redetermined. In vitro cumulative glutathione delivery across the bovine cornea was measured over 8 hours at $32^{\circ} \mathrm{C}$ in Franz diffusion cells. Carbopolcontaining Poloxamer systems exhibited shear-thinning behavior desirable for ocular formulations whilst Polyvinyl alcohol (PVA) and Polyacrylic acid (PAA) systems exhibited Newtonian behavior. Of the glutathione-containing systems, $0.2 \% \mathrm{w} / \mathrm{v}$ Carbopol 1342 was the most viscous with a viscosity of $960 \mathrm{cP}$ at a shear rate of $100 \mathrm{sec}^{-1}$. All formulations significantly increased the amount of glutathione delivered across the cornea (relative to an aqueous solution of glutathione) with the exception of $0.1 \% \mathrm{w} / \mathrm{v}$ Carbopol $940(p=0.12)$. Formulations containing $0.1 \% \mathrm{w} / \mathrm{v}$ Carbopol 934, $0.1 \%$ w/v Carbopol 1342 and $0.2 \%$ w/v Carbopol 940 improved penetration dramatically (ca. 30\%); but were not significantly different from each other. Therefore, Carbopol and Poloxamer formulations demonstrated enhanced penetration of glutathione across the cornea. The $0.2 \%$ w/v C940-containing-Poloxamer formulation was determined to be the most promising drug delivery system for ocular delivery of glutathione.
\end{abstract}

Keywords: Glutathione; Hydrogel; Ocular delivery; Rheology; Newtonian behavior; Sustained release; Permeation

\section{Introduction}

Glutathione ( $\gamma$-glutamyl-cysteinyl-glycine; GSH) is a highly hydrophilic thiol tri-peptide with a low-molecular weight of 307.4 $\mathrm{Da}[1,2]$. The reduced form of GSH serves as a strong intracellular antioxidant and plays an important role in the protection of the eye from oxidative stress $[1,3,4]$. In addition to its antioxidant activity, GSH serves as a cofactor of enzymes involved in the degradation of peroxides, such as GSH peroxidises. Moreover, as a component of $\mathrm{NADPH}$ pathway, it prevents cell components from being oxidized thus promoting DNA regulation and protein synthesis [3]. Franco et al. also reported the role of GSH in the regulation of cell-cycle, signal transduction and immunoresponse [5]. Given that all ocular tissues contain GSH as a primary antioxidant, replenishing the amounts of GSH in the eye could reduce the incidence of ocular diseases related to oxidative stress and aging, such as cataracts. The cornea is particularly susceptible to chemical insults and damage because it is the outermost layer of the eye and therefore exposed to the harsh environment. GSH maintains normal hydration, protects the cellular membrane and degrades xenobiotic agents in the cornea [4]. Therefore, GSH may play a role in the treatment of keratitis and other corneal diseases [4].

Due to anatomical and physiological characteristics, the eye is uniquely shielded and a highly protected organ that presents many barriers to effective ocular drug delivery [6]. The main physical barriers shielding the eye are tear film and cornea, which protect the anterior of the eye, Tear film is a protective layer that prevents the entry of foreign molecules into the eye. The blood-retina barrier, which protects the back of the eye [7]. The cornea is the main barrier against drug penetration to the inner tissues of the eye due to its small surface area and relative impermeability [8]. In addition, many ocular enzymes in the cornea could degrade GSH and prevent its therapeutic effect. These enzymes include endopeptidases (plasmin, collagenase) and exopeptidases, such as the hydrolytic aminopeptidase, which degrade amino acids [9].
The main advantages of topical administration are its ease and convenience of use and localized drug effects, thus, reducing systemic absorption and avoiding enzymatic degradation through hepatic first pass metabolism [10]. However, the natural ocular defense mechanisms lead to poor drug bioavailability. Blinking increases the production of the tear film's aqueous layer which in turn increases tear turnover to approximately $1 \mu \mathrm{L} / \mathrm{min}$, which is equivalent to $16 \%$ of the tear film turnover [11]. Both the removal of excess fluid and tear turnover reduce the extent of drug absorption. Conventional eye drops require frequent administration, with each drop being more than $30 \mu \mathrm{l}$, which is estimated to be the maximum volume the eye can accommodate without overflow. Drainage of instilled solution occurs within 1530 seconds after administration [12], therefore the majority of the drugs administered are removed before absorption. Although ocular ointments have the viscosity to increase pre-ocular drug retention time, they often cause irritability to the eye, resulting in lower compliance. In addition, their oily medium is not compatible with water soluble drugs such as GSH. Consequently, an ocular formulation with desirable characteristics that shows promising potential would be required to effectively deliver GSH. This delivery system should prevent enzymatic degradation of GSH, enhance its pre-corneal retention and penetration.

Hydrogels can be formed by dispersing polymers in an aqueous medium where they undergo swelling to produce a viscous gel capable of increasing pre-corneal retention time, prolonging contact time by increasing mucoadhesion and controlling drug release [12]. These properties increase trans-corneal absorption and hence the amount of drug reaching the anterior chamber of the eye. Poloxamers are nonionic triblock copolymers comprised of ethylene oxide and propylene oxide, which are non-irritating to the ocular surface [13]. With favorable characteristics such as strong hydrogen-bonding, high $\mathrm{Mw}$ and sufficient flexibility to interact with the mucus network contribute to excellent mucoadhesion. Another defining characteristic include their ability to increased stability of drugs, Carbopol is a synthetic high Mw polymer comprised of acrylic acid that are cross-linked with either allyl ether of pentaerythritol or allyl sucrose [3]. They are anionic and therefore offer increased mucoadhesion hence contact time [14]. In 







